\title{
Desafíos sociales y educación inclusiva
}

Pág. 80- 106

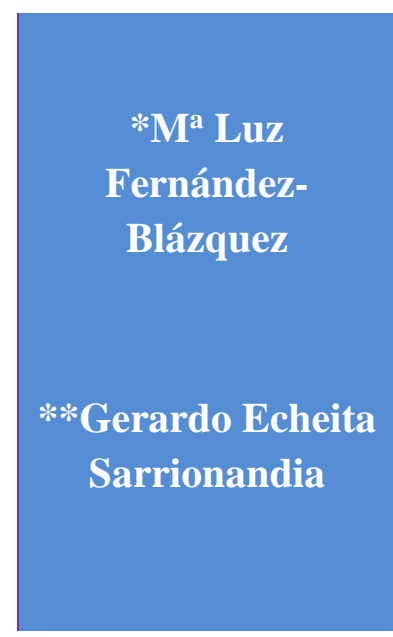

Universidad Autónoma de Madrid

Facultad de Psicología Departamento interfacultativo de

Psicología Evolutiva y de Educación

*mluz.fernandez@uam.es

**gerardo.echeita@uam.es

Fecha de entrega:

7 de julio

Fecha de aceptación: 10 octubre

\section{RESUMEN}

En este texto abordamos algunos de los principales desafíos de la sociedad moderna en relación con el desarrollo de la educación inclusiva y que remiten a esferas fundamentales del ser humano: a lo material, a lo relacional y a lo valorativo o moral. Respectivamente los denominamos: el desafío tecnológico, el desafío relacional: con las personas y con la Tierra, y el desafío ético. En el contexto social descrito, la escuela ha de tomar posición. Desde su comprensión como educación inclusiva opta por situar en el horizonte valores propios de la democracia y la equidad, y apuesta por el desarrollo de un proyecto social transformador, desarrollando el proyecto inclusivo en los centros escolares. Se suma así al movimiento social que reconoce la igual dignidad de todas las personas. Seguidamente centramos el análisis en aspectos que atañen a la conceptualización de la educación inclusiva y su puesta en acción: el modo en que se ha comprendido la diversidad humana. Nos centramos en dos grandes perspectivas desde las que se ha abordado y se aborda desde el sistema educativo la diversidad del alumnado: la perspectiva médica o esencialista y la perspectiva social o inclusiva y respondemos a varios interrogantes.

Palabras clave: educación inclusiva, desafíos sociales, diversidad, perspectiva inclusiva, perspectiva médica 


\title{
Social challenges and inclusive education
}

\begin{abstract}
In this text we address some of the main challenges of modern society in relation to the development of inclusive education and that refer to fundamental spheres of the human being: the material, the relational and the evaluative or moral. Respectively denominated as: the technological challenge, the relational challenge: with the Earth and the people, and the ethical challenge. In the social context described, the school has to take a stand. From its understanding as inclusive education, it opts to place the values of democracy and equity on the horizon, and bets on the development of a transformative social project, developing the inclusive project in schools. Thus, it joins the social movement that recognizes the equal dignity of all people. We then focus our analysis on aspects that concern the conceptualization of inclusive education and its implementation: the way in which human diversity has been understood. We focus on two main perspectives from which the diversity of students has been and is being addressed from the educational system: the medical or essentialist perspective and the social or inclusive perspective, and we answer several questions.
\end{abstract}

Keywords: Inclusive education, social challenges, diversity, inclusive perspective, medical perspective. 


\section{INTRODUCCIÓN}

El término educación inclusiva está conformado por dos palabras. El nombre común educación se complementa con el adjetivo inclusiva (Echeita, 2017). Esta construcción frente a formulaciones alternativas nos advierte que la cuestión básica que nos convoca afecta a la naturaleza de la Educación. Reflexionar sobre Educación no está al margen del contexto sociocultural donde esta se desarrolla (UNESCO, 2015). Específicamente en educación formal, educación y sociedad están estrecha e intencionalmente conectadas. Desde la función socializadora de la escuela, ${ }^{\mathbf{i}}$ cada generación debe definir la organización y los objetivos de la educación formal a fin de garantizar que la generación siguiente alcance el mayor grado de libertad y racionalidad posible para desenvolverse en la sociedad (Bruner, 1999). Por tanto, la educación escolar está inserta en un contexto del que no puede desligarse, y que le afecta.

Además de ser un instrumento social para transmitir el bagaje cultural a las nuevas generaciones, la educación se entiende como instrumento social que contribuye a la transformación de la sociedad. Es internacionalmente reconocida como herramienta para solucionar los desequilibrios sociales (PNUD, 2018; UNESCO/BIE, 2008; UNESCO, 2018). Desde esta función, la reflexión sobre educación se fundamenta en influencias políticas e ideológicas (Anastasiou, Kauffman y Di Nouvo, 2015; Black-Hawkins, Florian y Rose, 2017; Freire, 2015; Giroux, 1990; Hakala y Leivo, 2017; Haug, 2017; McLaren, 2005; Powell, Edelstein y Blanck , 2015; Tedesco, 2010) y es desde su posicionamiento dentro del contexto sociocultural en que se inserta, desde donde pone condiciones para la creación de determinado proyecto social.

Siguiendo con la argumentación iniciada, cuando al nombre educación le añadimos el adjetivo inclusiva, nos situamos ante un horizonte concreto de transformación: una educación que busca desarrollar un proyecto social inclusivo (UNESCO, 2020), que busca desarrollar los valores propios de la equidad y la justicia social (Norwich, 2014). Diversos estudios previos también sitúan la reflexión sobre educación inclusiva en este marco social amplio (Sapon-Shevin, 2013; Slee, 2019), más allá de lo que acontece en las propias escuelas, como apunta la ecología de la equidad (Ainscow, Dyson, Goldrick y West, 2013). Podemos señalar que, aún hoy, desarrollar 
una educación inclusiva continúa siendo una agenda pendiente (Echeita, 2019). Diversas causas pueden estar en la base de la compleja puesta en acción (Göransson y Nilholm, 2014; Messiou, 2016).

En este artículo nos centraremos en profundizar acerca de la interdependencia entre educación y sociedad. Para ello, abordaremos algunos de los que hemos identificado como principales desafíos sociales en el siglo XXI y que influyen en la puesta en acción de la educación inclusiva. Estos desafíos remiten a esferas fundamentales del ser humano: a lo material, a lo relacional y a lo moral. Así, mostraremos respectivamente: el desafío tecnológico, el desafío relacional: con la Tierra y con las personas, y el desafío ético. Seguidamente abordamos el papel que puede tener o tiene la educación inclusiva en el marco social descrito. Para finalizar, nos centraremos específicamente en cuestiones que atañen a la conceptualización de la educación inclusiva y su puesta en acción: el modo en que se ha comprendido la diversidad humana. Nos centraremos en dos grandes perspectivas desde las que se ha abordado y se aborda desde el sistema educativo la diversidad del alumnado: la perspectiva médica o esencialista y la perspectiva social o inclusiva y responderemos a varios interrogantes o hipótesis: ¿Qué se entiende como diversidad y Cómo se construye la diferencia?, ¿Qué valoración se hace de la diversidad?„¿A quién y cómo se ajusta la ayuda educativa?, ¿Quién puede realizar estos ajustes en la ayuda educativa? y ¿Qué papel tiene el docente? Para finalizar aportamos algunas conclusiones a modo de cierre.

\section{Desafíos sociales}

Vivimos en un mundo complejo (Morín, 2004). Son muchos los desafíos que implican esta complejidad y tienen diferente naturaleza y alcance. De los posibles desafíos actuales resaltaremos tres que, consideramos, remiten a esferas fundamentales del ser humano: a lo material, a lo relacional y a lo moral. Nos centraremos en tres por el alcance que tienen en nuestra actual sociedad y por las implicaciones que de una $\mathrm{u}$ otra forma tienen en las instituciones educativas. Los hemos denominado: el desafío tecnológico, el desafío relacional: con la Tierra y entre las personas, y el desafío ético. ${ }^{2}$ 


\section{El desafío tecnológico}

El imparable avance tecnológico, de ritmo vertiginoso y con destinos insospechados, se erige como una de las características idiosincrásicas de la época actual (PNUD, 2018). Su alcance afecta directa o indirectamente a la vida de todas las personas. Los avances técnicos tienen el poder de modificar y amplificar nuestras competencias como especie (Bruner, 1999), transformando nuestro modo de estar y relacionarnos con el mundo.

Siguiendo a Gil Vila (2016) podemos identificar que una de las principales consecuencias del desarrollo tecnológico es la transformación del tiempo y el espacio. La globalización, pareja al desarrollo técnico, acerca lo lejano y crea nuevos conocimientos. En este momento, la presencia no significa proximidad. Podemos estar en contacto de forma inmediata con personas a miles de kilómetros de distancia mediante imagen y sonido en tiempo casi real y tenemos la posibilidad de acceder a información procedente de otros contextos, convirtiendo en familiares situaciones vitales de personas que habitan lugares lejanos, física y culturalmente, acercándonos así a contextos y vidas diametralmente opuestos a los nuestros. Habitamos, pues, un mundo global donde el concepto de proximidad se ha ampliado y desligado de lo físico (Calderón, 2014). A través de las diferentes tecnologías de la información y la comunicación vidas lejanas llegan hasta nosotros de forma inmediata.

Nuestro control sobre el mundo físico a través de la movilidad también se ha transformado. Mediante los avances en la red de transporte podemos viajar hasta casi cualquier lugar de la tierra y conocer presencialmente esas formas de vida que identificábamos como opuestas o diferentes a las nuestras. Nuestro planeta, por tanto, se nos hace más accesible y manejable, e incluso nuestras posibilidades de conocimiento y movilidad exceden el propio planeta.

La tecnología, en simbiosis con nuestra vida (Morin, 2004), encierra también el riesgo de desestabilizar la configuración de la sociedad y de nuestra propia vida tal como la hemos conocido hasta ahora. A día de hoy, ante la situación de emergencia social mundial que vivimos debido a la COVID-19, se hace aún más palpable que nunca la brecha digital que 
continúa ampliándose entre quienes tienen acceso a la tecnología y quienes no (PNUD, 2018; UNESCO, 2015), con los altísimos niveles de desigualdad social que conlleva, entre otras cuestiones, el acceso o no a las posibilidades técnicas. Además, la manipulación de la biología posibilitada por los avances técnicos, o la propia competición entre la tecnología y la persona, son algunos de los desafíos indiscutibles donde este avance nos sitúa. Como ha dicho Harari (2017, p. 349): “¿Qué harán los humanos conscientes cuando tengamos algoritmos no conscientes y muy inteligentes capaces de hacer casi todo mejor?”.

Este gran desarrollo tecnológico también lleva parejo una transformación en el modo de habitar la Tierra. Si bien la tecnología está en simbiosis con nuestras vidas, no lo estamos así nosotros como especie con nuestro planeta. El avance tecnológico posibilita y muchas veces se sostiene desde la explotación indiscriminada de los recursos de la biosfera (UNESCO, 2017), a pesar de que en ese mismo avance algunos albergan parte de la esperanza para la solución (UNESCO, 2015). Todo este panorama va parejo también al crecimiento de los valores neoliberales y al surgimiento de nuevos modos de comprender y valorar la relación entre las personas, que nos conecta con el siguiente de los desafíos: nuestra relación con las personas y con el planeta.

\section{El desafío relacional}

La forma de vida actual también implica una transformación en los modos de relacionarnos: entre personas y con la propia Tierra. Comenzaremos con el desafío relacional con las personas y posteriormente nos centraremos en nuestra relación con el planeta, para abordar el tema de la sostenibilidad.

\section{- El desafío relacional con las personas}

A pesar de que:

...es la renuncia al egoísmo absoluto que exige el vivir en una comunidad lo que le proporciona [a la especie humana] su ventaja de especie dominante. La 
civilización moderna se ha hecho tan compleja, que hemos llegado a perder de vista esta sencilla idea que está en su fundación (Gil Vila, 2016, p. 35).

Diferentes situaciones ponen condiciones para que esto sea así, a la vez que lo visibilizan.

La sociedad moderna encierra en sí una revalorización del individuo frente a la colectividad (Castel, 2015; Gergen, 2015). La forma de vida actual nos lleva a cuestionar el vínculo de solidaridad con el otro tradicionalmente garantizado y valorado como positivo (Bauman, 2009; Castel, 2015; Gil Vila, 2016; Morin, 2004). En palabras de Bauman (2009): “(la) moderna razón líquida ve opresión en los compromisos duraderos; los vínculos durables despiertan su sospecha de una dependencia paralizante" (p. 70).

Además, los valores neoliberales que imperan en los países con alto desarrollo económico implican un estilo de vida en el que el progreso individual y la meritocracia se sitúan en la cúspide del ideal de progreso humano (McLaren, 2005; Owen, 2012; Sandel, 2020). Estos valores no contribuyen a la construcción de una ciudadanía donde la conciencia global y el compromiso desde la igualdad con el otro y con el entorno sean valores fundamentales. Este asunto ha sido estudiado desde diversas corrientes teóricas. Concretamente el feminismo asimila los valores neoliberales con el patriarcado, tradicionalmente asumido por el hombre, y reclama que, para la construcción de una verdadera democracia, debemos revalorizar el papel de las relaciones interpersonales basadas en el ideal de igualdad y en el reconocimiento de la interdependencia como lo característico, necesario y deseable en el desarrollo del ser humano (Tronto, 1987; 2017; Gilligan, 2013). El discurso descrito ha sido reconocido como predominante en las voces de las mujeres y circunscrito al ámbito de lo privado y familiar (Gilligan, 2013).

Añadido al cuestionamiento de los vínculos tradicionalmente garantizados y al avance de los valores neoliberales, los conflictos bélicos a día de hoy continúan generando exclusión y muerte. Aunque han experimentado una reducción, continúan surgiendo nuevos conflictos y otros tantos permanecen (PNUD, 2018).

REVISTA ANUAL, ACCIÓN Y REFLEXIÓN EDUCATIVA, Nº 46

Enero, 2021

ISSN L 2644-3775 
En todo este panorama, encontramos también que la misma tecnología que nos acerca el mundo y las personas, nos aleja de ellos. Desde la misma cotidianidad en que lo alejado se nos acerca, podemos volvernos insensibles a lo que vemos. Según Gil Vila (2016), llegamos a naturalizar las catástrofes humanitarias que diariamente vemos en los diferentes medios de comunicación, matando nuestra sensibilidad hacia el otro. Pese a encontrarnos en un momento en que todo está conectado, cuesta más que nunca visibilizar la necesaria y valiosa interdependencia positiva con los otros, que nos ha llevado como especie hasta el lugar en el que estamos. Según Gil Vila (2016), hay falta de predisposición en el siglo XXI para reflexionar sobre la parte de responsabilidad que nos toca con el sufrimiento ajeno.

Lo descrito hasta aquí encierra un enorme desafío: desde la situación perfilada, valores como la solidaridad, el cuidado o la igualdad, no quedan naturalizados en nuestro contexto. De hecho, para algunos, el propio funcionamiento de la moderna sociedad requiere de una distribución desigual de las oportunidades entre los grupos sociales (Owen, 2012; Moriña, 2010). Este tema conecta con el desafío que abordaremos con posterioridad: el desafío ético. Antes nos detendremos en el que hemos identificado como otro de los pilares del desafío relacional: nuestra relación con el planeta Tierra.

\section{-. El desafío relacional con la Tierra}

Si, como decíamos, la renuncia al egoísmo absoluto que requiere la vida en comunidad nos ha llevado como especie al lugar en el que estamos, también lo ha sido el modo de habitar nuestro planeta. Sin embargo, la relación entre los seres humanos y la Tierra desde este marco de desarrollo global e imparable, característico de la moderna sociedad, se construye en gran parte desde la explotación indiscriminada de los recursos naturales (UNESCO, 2017). La sostenibilidad del planeta es una de las mayores preocupaciones actuales: el cambio climático (World Meteorological Organization, 2019) o el agotamiento de recursos de la biosfera (UNESCO, 2017), son algunas de las consecuencias que hemos generado desde nuestra relación con la Tierra y a las que también hemos de hacer frente. 
Desde diferentes contextos internacionales se llama la atención sobre la necesidad de repensar el modo en que habitamos el mundo (UNESCO, 2017; PNUD, 2018); tal es el caso de los Objetivos de Desarrollo Sostenible 2030 (UNESCO, 2015; 2016). Parece que "el progreso de hoy se logra a costa de nuestros hijos. El cambio climático, la disminución masiva de la biodiversidad y el agotamiento de los recursos de la tierra y el agua dulce plantean graves amenazas para la humanidad" (Steiner, 2018, p. iii). Identificar el progreso de hoy desde el sacrificio de la humanidad futura, problematiza no solo el presente, sino también el porvenir.

El desafío, por tanto, es conseguir generar una relación sostenible con la Tierra y asumirnos como parte de ella, otorgándole el carácter matricial que tiene y ha tenido para nosotros como especie:

La Tierra no es la suma de un planeta físico, de una biosfera y de la humanidad. La Tierra es una compleja totalidad físico-biológica-antropológica, donde la vida es una emergencia de la historia de la Tierra y el hombre una emergencia de la historia de la vida terrestre. La relación del hombre con la naturaleza no puede ser concebida de modo reductor ni de manera separada. La humanidad es una entidad planetaria y biosférica (Morin, 2004, pp. 49-50).

Por ello es que, visibilizar la Tierra desde su carácter matricial y nuestro surgimiento como especie dentro de su historia y como parte de la historia de la vida terrestre, permite situarnos como pieza de una historia global. Desde lo creado nos pone en relación con el presente en una posición de actores: somos parte de una creación colectiva. La posición tomada en esa obra colectiva y viva que puede ser la humanidad, conecta con el último de los desafíos propuestos: el desafío ético.

\section{El desafío ético}

Probablemente uno de los mayores retos que tenemos actualmente sea el desafío ético. Ante ese imparable avance tecnológico, ante el desafío que alberga la sostenibilidad del planeta, ante los cambios en los modos de relacionarnos entre las personas, y ante el crecimiento de los valores 
neoliberales... ¿Qué hacemos? Este desafío nos sitúa como actores y actrices en la construcción del mundo que queremos. Como sociedad contamos con construcciones sociales, en forma de marcos jurídicos, que tratan de velar para reducir las desigualdades sociales y los procesos de exclusión tan presentes en nuestro mundo y basados en cuestiones no naturales (Castel, 2015).

Durante los últimos cincuenta años, ha habido progresos como resultado de las luchas por la igualdad y en contra de exclusión que viven grandes masas de la población mundial, como mujeres, minorías étnicas, personas en situación de discapacidad, personas refugiadas, personas mayores, o habitantes de lugares en conflicto (Slee, 2019). Cada situación de discriminación e injustica tiene detrás su propia historia de lucha y emancipación, como colectivo y como individuo, con características e hitos particulares (Sapon-Shevin, 2013). Seguramente podemos situar hacia los años 60 y 70 del siglo XX, el comienzo de un fuerte movimiento hacia la igualdad de derechos para todas las personas. Tal es el caso de la lucha de los derechos civiles de los hombres y mujeres negras en EE.UU contra el racismo imperante en su sociedad, el crecimiento del movimiento para la liberación de la mujer o, entre otras, las llamadas políticas de integración para las personas consideradas con discapacidad y que recientemente se han integrado también en el marco de los derechos humanos (Naciones Unidas, 2006). El Informe sobre Desarrollo Humano de 2018 (PNUD, 2018) muestra que desde 1990 a 2017 ha habido una reducción a nivel mundial del grupo de personas que se sitúa en el índice de desarrollo humano bajo y se ha triplicado el número de personas que lo hace en el índice de desarrollo humano alto y muy alto. Pero, como analiza Gila Vila (2016), aún hay mucho camino por recorrer:

Una de las paradojas más trágicas de nuestro tiempo: ahora que tenemos más derechos, ahora que contamos con una Declaración de Derechos Humanos que se ha ido desarrollando poco a poco en los últimos sesenta años en sus distintos ámbitos, es cuando más nos alejamos de ella, cuando más la vulneramos, dando lugar a un sinfín de situaciones de desigualdad y exclusión social caracterizadas por un denominador común: el sufrimiento (p. 24). 
La exclusión social llama a la exclusión educativa y viceversa (Echeita, 2013; Etxebarria, 2018; Gil Vila, 2016; Moriña, 2010; Murillo y Martínez-Garrido, 2017), es una espiral que mina la dignidad y la paz (UNESCO, 2015). Por tanto, este es el mayor desafío: cómo posicionarnos para contribuir en el avance hacia una sociedad de todos, con todos y sostenible. En este contexto: ¿cómo podemos situar la educación inclusiva?

\section{Desafíos sociales y educación inclusiva}

Hasta aquí hemos identificado y compartido brevemente tres grandes desafíos sociales y que se interrelacionan: el desafío tecnológico, el desafío relacional: con la Tierra y entre las personas, y el desafío ético. Apuntábamos que todos ellos tienen implicaciones en la manera de conceptualizar, organizar y desarrollar lo que acontece en la escuela. Así, el panorama descrito puede llevarnos a pensar que una educación distinta, una educación más justa e inclusiva no surgirá de forma natural a partir del orden social existente (Göransson y Nilholm, 2014; Tedesco, 2004), sino como el resultado de acciones conscientes, voluntarias y reflexivas que requieren mucho más esfuerzo, tanto cognitivo como emocional, que el exigido por la ideología dominante.

Por tanto, esto nos sitúa en el otro de los pilares con los que comenzamos este ensayo y en estrecha relación con el último de los desafíos descritos: el necesario posicionamiento de la educación escolar ante la sociedad. Ante este contexto, qué puede hacer la educación escolar. Tiene que elegir: ¿contribuir a la reproducción social (Bordieau, 1997; Lahire, 2003), o posicionarse como herramienta para la transformación (Freire, 2009; Giroux, 1990; McLaren, 2005)? La educación escolar asumida ahora como educación inclusiva se sitúa desde esta segunda opción: como actriz clave para la transformación social, como transformadora de los desequilibrios sociales (Calderón y Echeita, 2016) y se alinea con la intención de ir poniendo condiciones favorables para la creación de una sociedad de todos y para todos. ${ }^{3}$ Es desde su posicionamiento en respuesta al desafío ético desde el que también cobrarán forma los otros dos desafíos. La ideología básica detrás de esta forma de entender la educación se relaciona con

REVISTA ANUAL, ACCIÓN Y REFLEXIÓN EDUCATIVA, Nº 46

Enero, 2021

ISSN L 2644-3775 
los valores democráticos y la justicia social (Norwich, 2014; Haug, 2017). Sitúa nuestras reflexiones y acciones en dialéctica con lo "excluyente" (Ainscow y West, 2006) y ofrece una perspectiva desde la que analizar y desarrollar los desafíos de la equidad en la educación escolar (Echeita, 2019).

Podemos decir que en los últimos 50 años ha habido un movimiento educativo, abarcando políticas y directrices internacionales que van hacia ese lugar de cambio (UNESCO/BIE, 2008; UNESCO, 2015; 2016; 2020). Es el caso de la agenda educativa 2030 que, con el Objetivo de Desarrollo Sostenible (ODS) número 4, busca: “Garantizar una educación inclusiva y equitativa de calidad y promover oportunidades de aprendizaje permanente para todos" (UNESCO, p.n. 2015).

A pesar de los avances, el reconocimiento de la igualdad entre las personas que implica el derecho a una educación de calidad con equidad, sigue sin estar garantizado en la escuela, y allí donde avanza aún hoy encontramos contradicciones y dilemas en su puesta en acción (Artiles y Kozleski, 2016; Azorín y Ainscow, 2018; Hakala y Leivo, 2017; Norwich, 2014). Este reconocimiento de la igualdad del alumnado ha estado y está vinculado al modo en que se ha comprendido la diversidad humana y a las acciones que se han puesto en funcionamiento para responder a ella. A continuación, profundizamos sobre esta cuestión que está de base en el modo de conceptualizar la educación inclusiva y su puesta en acción.

\section{¿La escuela frente a la diversidad?}

Las personas son iguales en la diversidad (Echeita, Simón, López y Urbina, 2014). Ser iguales en la diversidad significa que todos los seres humanos tienen en común el propio hecho de ser distintos y que lo único en lo que son iguales es en su igual dignidad. Esta creencia es contraria a posturas tradicionales enraizadas en nuestra sociedad, ligadas a las ideas de normalidad y anormalidad (Sapon-Shevin, 2013). Tradicionalmente la idea de persona normal quedaba encarnada en ser hombre, blanco, “capaz”, de clase media y occidental (Pfeiffer, 2000 citado 
en Calderón, 2014). Desde estas creencias, aquello que se salía de la norma era considerado como anormal y en la mayoría de las ocasiones convertido en patológico, desde una valoración negativa hacia la diversidad. La perspectiva descrita puede decirse que asienta sus bases en una concepción biológica del ser humano (Calderón, 2014). Según este mismo autor, frente a esta visión sobre la diversidad humana encontramos una alternativa, que se acerca a una comprensión del ser humano como diferencia. El autor relaciona la diversidad entre las personas con el grado de apertura del ser humano, que se amplía más allá de lo biológicamente dado, como ser histórico e inacabado. Podemos decir, por tanto, en palabras de Calderón:

Lo común a todos los seres humanos es precisamente lo diferente: no hay un ser humano igual a otro, no hay un ser humano que no cambie. Cada hombre o mujer tiene una vida, una biografía. El ser humano, por tanto, es diferente de sí mismo y de los demás $(2014$, p. 41).

Encontramos así la asunción de la diversidad como característica inherente de la humanidad. Esta perspectiva cuestiona la visión sobre la diversidad descrita con anterioridad enraizada en la biología y generalmente presentada de modo binario: normal-anormal o capaz-incapaz (Echeita y Fernández-Blázquez, 2017).

Las creencias identificadas hasta aquí se construyen y mantienen desde las diferentes instituciones sociales y por tanto también desde la escuela. Una de sus posibles traducciones dentro del contexto escolar la podemos identificar desde el modo en que se ha comprendido la diversidad presente en el alumnado y cómo se ha configurado la respuesta a esta diversidad. Profundizar en estas cuestiones es el principal objetivo de los siguientes epígrafes.

\section{- Perspectivas sobre la diversidad en educación}

A lo largo de la historia podemos identificar dos grandes perspectivas en torno a las que aglutinar las respuestas ofrecidas desde el sistema educativo a la diversidad presente en el alumnado: la perspectiva individual, médica o esencialista y la perspectiva social o inclusiva. La noción de perspectiva nos ayuda a explicar un hecho, resaltando qué vemos, cómo lo interpretamos y cómo actuamos sobre él. Puede ser similar a la idea de modelo o paradigma 
(Ainscow y Echeita, 2011). A continuación, vamos a compartir lo característicos de ambas perspectivas desde la respuesta a cinco grandes interrogantes: a) ¿Qué se entiende como diversidad y cómo se construye la diferencia?, b) ¿Qué valoración se hace de la diversidad?, c) ¿quién puede realizar los ajustes en la ayuda educativa?, d) ¿A quién y cómo se ajusta la ayuda educativa? y e) ¿Qué papel tiene el docente? Nos apoyaremos para ello en elaboraciones previas sobre la respuesta ofrecida desde el sistema educativo a la diversidad del alumnado (Echeita, 2013; Echeita y Fernández-Blázquez, 2017) y algunas reflexiones acerca del origen de la diversidad humana (Calderón, 2014; Gil Vila, 2016; Hunt y Sullivan, 1974, citado en Coll y Miras, 2001). ${ }^{4}$

\section{- La perspectiva médica o remedial o la perspectiva individual o esencialista}

Ha sido dominante en la forma de pensar y responder a la diversidad del alumnado en la escuela desde mediados del siglo XX. Ha supuesto, entre otras cuestiones, la invisibilidad social de niños y niñas considerados especiales, desde el mantenimiento del sistema de educación de doble vía. Lleva implícita la creencia de que el sistema educativo ordinario no precisa transformar sus prácticas para responder a determinado alumnado y que el alumnado que hay en sus aulas no tiene diversidad. De forma específica y respondiendo a los cinco interrogantes planteados, en la perspectiva médica o individual:

- ¿Qué se entiende como diversidad y cómo se construye la diferencia? Desde la perspectiva médica o esencialista se generan diferencias en bases a criterios predefinidos a priori, cobrando gran peso en esta configuración el papel de lo biológico, por ejemplo, el ritmo o el nivel de desarrollo evolutivo en comparación con el nivel de desarrollo considerado normotípico. La revalorización de lo biológico se materializa también en el hecho de que los problemas o dificultades presentes en el desarrollo del proceso educativo se entienden como parte de una limitación o deficiencia interna al alumnado. Podríamos hablar también de una concepción estática de las diferencias individuales (Hunt y Sullivan, 1974, citado en Coll y Miras, 2001). Desde este posicionamiento se considera que se puede y se debe identificar a los alumnos que son 
diferentes en relación al criterio de diversidad predefinido y, por tanto, se desarrollan acciones que buscan categorizar al estudiante en torno a esas diferencias individuales de origen interno y que le sitúan fuera de lo considerado normal.

- ¿Qué valoración se hace de la diversidad? Las diferencias son constituidas como problema en el desarrollo de la educación escolar porque dificultan el desarrollo del proceso educativo de los normales (que viene predefinido). Por tanto, el alumno raro es quien tiene algo mal que interfiere en el proceso de aprendizaje y necesita ser arreglado. Esto supone que las actividades educativas principalmente se dirigen a paliar los déficits cognitivos, sensoriales o motores identificados a fin de lograr el ajuste de ese alumnado al alumnado considerado normal, frente a otras posibilidades, como el desarrollo de su potencial personal o el desarrollo de un currículo ajustado al alumnado. En muchas ocasiones cuando no se consigue paliar la dificultad que se ha generado, la diferencia es eliminada, tema que conecta con el siguiente epígrafe.

- ¿A quién y cómo se ajusta la ayuda educativa? Solo el grupo de alumnos categorizados como diferentes, raros, distintos o especiales requiere un ajuste especial en la ayuda educativa. Una consecuencia práctica y supuestamente lógica de esta creencia es la organización de la educación con dos o varias vías diferenciadas: una escuela normal para el alumnado normal y una escuela o diferentes dispositivos especiales para el alumnado etiquetado como diferente. Esta escuela, grupo o programas especiales, genéricamente hablando, ordena, organiza y aglutina las ayudas especiales o compensatorias que pudieran ser apoyo para el alumnado (profesorado, medios técnicos, instalaciones) y únicamente están disponibles para el alumnado considerado igualmente especial, “diverso”. Las ayudas especiales para el alumnado especial se prestan mejor en grupos de alumnos homogeneizados en base a determinadas características, por ejemplo, discapacidad, género, grupo cultural o clase social (Parrillas, 2002). En el caso del alumnado con discapacidad, las ayudas especiales se concentran en escuelas o programas especiales diferenciados para aquellos a quienes se considera posible ayudar y para el resto existen otros centros específicos. 
- ¿Quién puede realizar estos ajustes en la ayuda educativa? Estas ayudas consideradas especiales precisan de profesorado especializado frente al profesorado ordinario. A modo de ejemplo para visibilizar esta circunstancia, podemos señalar que en España continuamos llamando profesorado de Pedagogía Terapéutica a aquellos expertos en dar respuesta a estas necesidades.

- ¿Qué papel tiene el docente? El docente desde esta perspectiva se sitúa ante un grupo de alumnos normales, desde una mirada que considera innecesario el ajuste en la ayuda educativa. Por tanto, el docente puede ser visto más bien como un profesional técnico que se limita a transmitir un curriculum con independencia del alumnado que conforme su grupo de aula (porque las diferencias han sido eliminadas). El tipo de práctica pedagógica que desde aquí desarrolla el docente es más congruente con el modelo transmisivo.

\section{- La perspectiva inclusiva}

La perspectiva que podríamos empezar a llamar inclusiva implica un giro copernicano respecto a lo expuesto hasta aquí en el modo de conceptualizar las diferencias entre personas y en la organización de las prácticas educativas. Supone asumir la igualdad en la diversidad (Echeita, Simón, López y Urbina, 2014). Niega que las dificultades de aprendizaje sean algo inherente a la persona y pone en valor la configuración del contexto y la organización de las ayudas educativas como mecanismos para facilitar o dificultar el desarrollo de cada aprendiz. Otorga, por tanto, un papel primordial a la comunidad educativa tanto en la configuración de las propias dificultades como, llegado el momento, en las soluciones para el desarrollo del proceso educativo. Respondiendo, ahora desde la perspectiva inclusiva, a los interrogantes inicialmente planteados:

¿Qué se entiende como diversidad y cómo se construye la diferencia? Se amplía el foco acerca de la diversidad humana dando cabida a otras fuentes además de las predefinidas y dejan de ser interpretadas de modo biologicista: capacidades, intereses, procedencia, o cualquier otra condición personal o social, y se amplía el foco hacia el contexto y la historia de la persona. La 
diversidad se considera inherente a todo alumno. El modo en que se constituye la diferencia también varía: se identifica poniendo en relación las características del alumnado con lo que sucede en el contexto, en este caso, con la práctica pedagógica. Así, encontramos una concepción interaccionista sobre el origen de la diversidad humana (Hunt y Sullivan, 1975 citado en Coll y Miras, 2001).

¿Qué valoración se hace de la diversidad? Frente a configurarse como hecho problemático, se constituye como fortaleza y lo característico del ser humano (Calderón, 2014; Echeita, 2014). Además, se entiende que la inclusión social se vive desde la inclusión escolar y viceversa (Echeita, 2014). Por ello, la escuela debe ser un lugar privilegiado para revalorizar la diferencia y para construir una sociedad incluyente y cohesionada. Desde la propia idea de diferencia, el alumnado puede crecer en la escuela como persona reconocida individualmente y, a su vez, la escuela puede contribuir hacia la construcción de la diferencia como rasgo positivo y distintivo de las personas, comenzando por el propio reconocimiento de esta.

¿A quién y cómo se ajusta la ayuda educativa? Desde esta mirada, las dificultades educativas se configuran como señales de alerta acerca de las prácticas docentes y organizaciones escolares erróneas. Se reconoce que algunas características del alumnado pueden ser más modificables que otras, pero se asume la educabilidad inherente a toda persona (Strauss y Ziv, 2004; Puig, 2012). Se reconoce que todo el alumnado que está en la escuela tiene derecho a aprender. Por tanto, la acción educativa debe diseñarse y desarrollarse pensando en la diversidad presente en el alumnado, de forma que sea accesible y pertinente para todos ellos. Los sistemas de apoyo, de carácter social, personal o de cualquier otra índole, están disponibles para todo el alumnado que lo precise, de forma temporal o permanente. Desde esta mirada se entiende el apoyo escolar como todo aquello que hace o puede hacer el centro para responder con equidad a la diversidad presente en el alumnado (Echeita, 2019). Incluye medidas organizativas, didácticas y van más allá de las respuestas educativas que puede dar el profesorado llamado de apoyo.

¿Quién puede realizar estos ajustes en la ayuda educativa? Todo el profesorado debe responder educativamente al progreso de todo el alumnado, sin exclusiones. El responsable de 
la educación de todo el alumnado es el docente regular del aula. Por tanto, no debe depositarse la responsabilidad del alumnado más desafiante, por ejemplo, el considerado con necesidades educativas especiales, siguiendo el ejemplo que hemos adoptado, en un profesorado especial o experto. El docente es el responsable de la organización de la respuesta educativa del aula donde esté desarrollando su labor. El considerado hasta ahora profesorado especial, y que puede contar con unos conocimientos y habilidades especializados, puede constituirse como apoyo al docente para organizar y coordinar las actuaciones docentes de modo que pueda garantizarse una respuesta educativa ajustada a las necesidades educativas de todo el alumnado. En Canadá, por ejemplo, ese perfil profesional es denominado Profesorado de Estudiantes y Recursos (Echeita et al, 2020).

¿Qué papel tiene el docente? Asumir la responsabilidad de diseñar y desarrollar prácticas pedagógicas diversificadas, ajustables a la realidad de todo el alumnado es una tarea compleja y sujeta a dilemas éticos (Echeita, 2013; Naiaran y Schlessinger, 2017, Harris, Carrington y Ainscow, 2018), que demanda a los docentes reflexionar sobre sus sistemas de prácticas (Puig, 2012) y saber responder a la tensión de la instrucción (Naiaran y Schlessinger, 2017). Responder a los dilemas no permite oscilaciones prescritas. Un rol docente bajo el que no se puede responder desde posiciones técnicas y descontextualizadas, enfatiza, entre otras cuestiones, el valor de la reflexión sobre la práctica docente. Implica, por ello, revalorizar la profesión docente como una profesión intelectual (Freire, 2009; Giroux, 1990; Schlessinger, 2018; Fernández-Batanero, 2013; Hakala y Leivo, 2017). Requiere de procesos de formación permanente en el profesorado (Arteaga y García, 2008) basados en un modelo reflexivo y no prescriptivo, que fomente un cambio en las concepciones y las prácticas docentes (Pozo, Loo y Martín, 2016), incidiendo en las creencias sobre diversidad (Moriña y Parrillas, 2006; Naiaran y Schlessinger, 2017), y siendo incentivado desde los planes de mejora e innovación escolar (Echeita, 2014).

Hasta aquí hemos delineado algunas creencias, conductas y valores que configuran las premisas básicas desde donde se está construyendo la nueva perspectiva de una educación inclusiva. A modo de síntesis, acabamos de perfilar dos de las grandes perspectivas que nos permiten 
visibilizar el modo en que se ha comprendido la diversidad presente en el alumnado y las respuestas organizativas, curriculares y profesionales que se han dado a esta. Desde la perspectiva médica o esencialista la diferencia es generada principalmente por criterios predefinidos con anterioridad y desde una mirada que convierte la dificultad en biológica. Sitúa en el interior del alumno la dificultad y la interpreta como inmutable. Esta se identifica como problemática, porque impide el ajuste del alumno al contexto escolar, que viene predefinido. Desde esta posición, el docente y la escuela no tienen mucho que hacer en la educación del alumnado que se sale del patrón considerado normal: el alumno es así.

Desde la perspectiva inclusiva la diversidad se asume como inherente a la humanidad y los parámetros contextuales y culturales forman parte de su configuración: motivaciones, origen sociocultural, capacidades, entre otras. Se asume que la respuesta de cada alumno dentro del proceso educativo es consecuencia de la interacción entre sus propias características y lo que le demanda la institución escolar. Desde esta comprensión, la escuela puede contribuir al proceso educativo de todo el alumnado. Esta perspectiva es todavía contrahegemónica a la esencialista o médica. La perspectiva inclusiva supone en sí mismo revalorizar el papel de la institución escolar como creadora de construcciones alternativas a la comprensión de la diversidad tradicionalmente sostenida y la asunción de un rol clave en el diseño de una educación que responda a todo el alumnado. De nuevo, enfatizamos, sitúa a la escuela como protagonista en la construcción de un proyecto social inclusivo, desde el que las diferencias entre las personas sean vistas como riqueza y del que todas las personas formen parte. Además, supone contribuir a garantizar el cumplimiento del derecho a la educación inclusiva (NN.UU, 2006). 


\section{CONCLUSIONES}

Hemos compartido el significado y sentido de lo que entendemos como educación inclusiva, en el contexto de algunos de los más importantes desafíos a los que se enfrentan las sociedades contemporáneas. Específicamente el que hemos denominado como desafío ético, sitúa a la escuela como parte del movimiento social desde el que buscamos el reconocimiento de la igual dignidad de todos los seres humanos, tiene un anclaje fundamental a nivel social por su carácter normativo en los derechos humanos universales. Este desafío se conecta profundamente con el papel que juega la educación en la sociedad actual donde determinadas diferencias naturales devienen en injustas desigualdades y desventajas sociales. Precisamente consideramos que la educación, entendida como una educación inclusiva, puede contribuir en la transformación del panorama de discriminación (educativa y social) que viven muchas personas por razones de género, salud, capacidad, procedencia u otras, según donde vivan.

Al profundizar, entonces, acerca de nuestra comprensión sobre la educación inclusiva, nos hemos detenido en identificar su estrecha relación con las perspectivas desde las que se comprende la diversidad del alumnado y se responde a ella en las aulas. En este sentido hemos descrito los elementos que sostendrían la perspectiva inclusiva, en contraste con aquellos que sostienen perspectivas no inclusivas y que históricamente han recibido denominaciones como perspectiva individual, remedial, esencialista o médica, según el punto de vista desde el cual han sido analizadas. También nos hemos hecho eco de algunas de las múltiples implicaciones que esta perspectiva inclusiva tendría en la práctica y de cuáles son las dimensiones, los procesos y algunos cambios que deben articularse con equidad para poder avanzar hacia las expectativas educativas y sociales en ella depositadas.

Todo ello nos ha hecho asumir y compartir los análisis que señalan la naturaleza ecológica en la tarea de llevar los valores inclusivos a la acción (dependiente de lo que acontece en los centros escolares, entre estos, es decir, en el sistema educativo y más allá de este), su carácter sistémico (dado que atañe a todos los elementos o componentes de un sistema educativo), pero también 
su urgente y necesaria concreción local (encarnada en una realidad singular y en un aquí y ahora propio de cada centro escolar). Esta multifacética realidad hace comprensible que, en no pocas ocasiones, se plantee que estamos ante una realidad mal definida o imprecisa y que también para algunos autores o agentes educativos, tenga un alcance limitado (por ejemplo, referido solo a cierto alumnado más vulnerable) o parcial (que atañe solo a reformas puntuales referidas, precisamente, a la atención a un alumnado considerado en términos genéricos como especial).

A este respecto hemos tratado de compartir nuestra visión sobre la educación inclusiva: más global, más sistémica y más ecológica y, por ello, aún por construir. Esto nos sitúa en la necesidad de continuar profundizando acerca de nuestra comprensión sobre los procesos que se vinculan a las reformas educativas, la mejora escolar, la innovación educativa y el cambio conceptual, habida cuenta de que en todos estos planos (respectivamente, el sistema en su conjunto, el centro escolar y lo que acontece en las aulas a tenor de cómo se piensa y se siente la acción educativa, Pozo, Loo y Martín, 2016), tienen que darse transformaciones profundas para que el desarrollo de una educación más inclusiva sea posible.

\section{REFERENCIAS}

Ainscow, M. y West, M. (2006). Improving Urban Schools. Leadership and collaboration. Berkshire: Open University Press.

Ainscow, M. y Echeita, G. (2011). La educación inclusiva como derecho. Marco de referencia y pautas de acción para el desarrollo de una revolución pendiente. Tejuelo, $\mathrm{n}^{\circ} 12$. pp. 26-46.

Ainscow, M., Dyson, A., Goldrick, S. y West, M. (2013). Promoviendo la equidad en educación. Revista de investigación en educación. 11(3), pp. 44-56. https://dialnet.unirioja.es/servlet/articulo? codigo $=4735222$ 
Anastasiou, D., Kauffman, J. M., y Di Nuovo, S. (2015). Inclusive education in Italy: description and reflections on full inclusion. European Journal of Special Needs Education, 30(4), pp. 429-443. Doi: 10.1080/08856257.2015.1060075/.

Arteaga Martínez, B. y García, M. (2008). La formación de competencias docentes para incorporar estrategias adaptativas en el aula. Revista complutense de educación, 19(2), pp. 253-274. http://revistas.ucm.es/index.php/RCED/article/view/RCED0808220253A.

Artiles, A.J., y Kozleski, E. B. (2016). Inclusive education's promises and trajectories: Critical notes about future research on a venerable idea. Education Policy Analysis Archives, 24(43), pp. 1-29. Doi.org/10.14507/epaa.24.1919/.

Azorín, C. y Ainscow, M. (2018). Guiding schools on their journey towards inclusion. International Journal of Inclusive Education, pp. 1-19. Doi: 10.1080/13603116.2018.1450900/.

Bauman, Z. (2009). Amor líquido. Acerca de la fragilidad de los vínculos humanos (5 ${ }^{\mathrm{a}}$ reimpr.). Madrid: Fondo de Cultura Económica de España.

Black-Hawkins, K., Florian, L. y Rouse, M. (2017). Achievement and Inclusion in Schools. New York: Routledge.

Bruner, J. (1999). La educación puerta de la cultura. Madrid: Visor.

Calderón, I. (2014). Educación y esperanza en las fronteras de la discapacidad. Estudio de caso único sobre la construcción creativa de la identidad. Madrid: CINCA.

Calderón, I. y Echeita, G. (2016). Desafíos ineludibles para la construcción de escuelas inclusivas. Dosier Graó, 1, pp. 35-41.

Castel, R. (2015). La inseguridad social: ¿Qué es estar protegido? (6 reimpr.). Buenos Aires: Ediciones Manantial.

Coll, C. y Miras, M. (2001). Diferencias individuales y atención a la diversidad en el aprendizaje escolar. En A. Marchesi, C. Coll y J. Palacios (Comp.) Desarrollo psicológico y educación (Tomo 2), pp. 331-353. Madrid: Alianza Editorial.

Coll, C. (2013). El currículo escolar en el marco de la nueva ecología del aprendizaje. Aula de $\begin{array}{lllll}\text { Innovación Educativa, } & 219, & \text { pp. } & \text { En }\end{array}$ http://diposit.ub.edu/dspace/bitstream/2445/53975/1/627963.pdf

Echeita, G. (2017). Educación inclusiva. Sonrisas y lágrimas. Aula abierta, 46, pp. 17-24. En https://www.unioviedo.es/reunido/index.php/AA/article/view/11982/.

Echeita, G. (2019). Educación inclusiva o el sueño de una noche de verano. Madrid: Octaedro. 
Echeita, G. (2013). Inclusión y exclusión Educativa. De nuevo “Voz y quebranto”. Revista electrónica Iberoamericana sobre calidad, eficacia y cambio en Educación. 11 (2), pp. 99-118. En http://www.redalyc.org/pdf/551/55127024005.pdf.

Echeita (2014). Educación para la inclusión o educación sin exclusiones. Bogotá: Narcea.

Echeita, G. y Fernández-Blázquez, M.L. (2017). El contexto educativo. En B. Gutierrez y Á. Brioso (coord.), Desarrollos diferentes, pp. 201-215. Madrid: Sanz y Torres.

Echeita, G., Simón, C., López, M. y Urbina, C. (2014). Educación inclusiva. Sistemas de referencia, coordenadas y vórtices de un proceso dilemático. En M.Á. Verdugo y R. Shalock (Coords.). Discapacidad e Inclusión. Manual para la docencia., pp. 307-328. Salamanca: Amaru.

Echeita, G. y Simón, C., (2020). El papel de los Centros de Educación Especial en el proceso hacia sistemas educativos más inclusivos. (Coords.). Madrid: Ministerio de Educación y Formación Profesional. Centro Nacional de Innovación e Investigación Educativa (CNIIE).

Fernández Batanero, J. M. (2013). Competencias docentes y educación inclusiva. Revista Electrónica de Investigación Educativa, 15(2), pp. 82-99. En http://redie.uabc.mx/vol15no2/contenido-fdzbatanero.html.

Freire, P. (2009). Pedagogía de la autonomía (11ºd.). Madrid: Siglo XXI.

Freire, P. (2015). La educación como práctica de la libertad. Madrid: Siglo XXI.

Gergen, K. (2015). El ser relacional. Más allá del Yo y de la Comunidad. Bilbao: Desclée de Brouwer.

Gil Vila, F. (2016). La sociedad vulnerable. Por una ciudadanía consciente de la exclusión y la inseguridad sociales. Madrid: Tecnos (Grupo Anaya).

Gilligan, C. (2013). La ética del cuidado. Madrid: Fundació Víctor Grífols i Lucas.

Giroux, H. (1990). Los profesores como intelectuales. Hacia una pedagogía crítica del aprendizaje. Barcelona: Centro de Publicaciones del Ministerio de Educación y Ciencia y Ediciones Paidós.

Göransson, K. y Nilholm C. (2014). Conceptual diversities and empirical shortcomings- a critical analysis of research on inclusive education. European Journal of Special Needs Education, 29(3), pp. 265-280. Doi.org/10.1080/08856257.2014.933545/.

Hakala, J. T., \& Leivo, M. (2017). Tensions in the New Millennium: Inclusion Ideology and Education Policy in the Finnish Comprehensive School. Journal of Education and Learning, 6(3), pp. 287-298. Doi:10.5539/jel.v6n3p287/. 
Harari, Y. N. (2017). Homo deus: Breve historia del mañana. España: Debate.

Haug, P. (2017). Understanding inclusive education: ideals and reality. Scandinavian Journal of Disability Research, 19(3), 206-217, doi.org/10.1080/15017419.2016.1224778/.

Lahire, B. (2003). Los orígenes de la desigualdad escolar. En Á. Marchesi y C. Hernández (eds.), El Fracaso escolar: Una perspectiva internacional, pp. 61- 71. Madrid: Alianza.

McLaren, P. (2005). La vida en las escuelas: una introducción a la pedagogía crítica en los fundamentos de la educación. Buenos Aires: Siglo XXI.

Messiou, K. (2016). Research in the field of inclusive education: time for a rethink? International Journal of Inclusive Education,21(2), pp. 146-159. Doi: 10.1080/13603116.2016.1223184/.

Morin, E. (2004). La mente bien ordenada: repensar la reforma, reformar el pensamiento $\left(6^{\mathrm{a}}\right.$ ed.). Barcelona: Seix Barral.

Moriña, A. (2010). Vulnerables al silencio. Historias escolares de jóvenes con discapacidad. Revista de educación, 35(3), pp. 667-690.

Moriña, A. y Parrilla, A. (2006). Criterios para la formación permanente del profesorado en el marco de la educación inclusiva. Revista de Educación, $n^{o}$ 339, pp. 517-539. En http://hdl.handle.net/11441/66853/.

Murillo, J. y Martínez-Garrido, C. (2017). Estimación de la magnitud de la segregación escolar en América Latina. Revista Internacional de Investigación en Educación, 9 (19), pp. 1130. Doi: 10.11144/Javeriana.m9-19.emse/.

Murillo, F.J. y Krichesky, G.J. (2015). Mejora de la escuela: Medio siglo de lecciones aprendidas. REICE. Revista Iberoamericana sobre Calidad, Eficacia y Cambio en Educación, 13(1), pp. 69-102.

Naciones Unidas (2006). Convención de las Naciones Unidas sobre los derechos de las personas con discapacidad. En https://www.un.org/esa/socdev/enable/documents/tccconvs.pdf.

Nairaian, S., y Schlessinger, S. (2017). When theory meets the "reality of reality": Reviewing the sufficiency of the social model of disability as a foundation for teacher preparation for inclusive education. Teacher Education Quarterly, 44(1), pp. 81-100. En https://www.jstor.org/stable/90003619?seq=1\#page_scan_tab_contents/.

Norwich, B. (2014). Changing policy and legislation and its effects on inclusive and special education: a perspective from England. British Journal of Special Education, 41(4), pp. 403-425. Doi: 10.1111/1467-8578.12079/. 
Owen, J. (2012). Chavs. La demonización de la clase obrera. Madrid: Capitán Swing.

Parrillas, A. (2002). Acerca del origen y el sentido de la educación inclusiva. Revista de Educación, 327, $\quad$ pp. $11-29 . \quad$ En https://dialnet.unirioja.es/servlet/articulo?codigo=246067/.

Pozo, I. Loo, C. y Martín, E. (2016). El cambio de las concepciones y las prácticas docentes como factor de cambio educativo. En J. Manzi, y M. R. García. (Eds.). Abriendo las puertas del aula: Transformación de las prácticas docentes, pp. 545-584. Santiago, Chile: Centro UC.

Powell, J., Edelstein, B. y Blanck, J. (2015). Awareness-raising, legitimation or backlash? Effects of the UN Convention on the Rights of Persons with Disabilities on education systems in Germany. Globalisation, Societies and Education, 14(2), pp. 227-250. Doi: 10.1080/14767724.2014.982076/.

Programa de Naciones Unidas para el Desarrollo (2018). Índices e indicadores del desarrollo humano. Actualización estadística 2018. Nueva York: PNUD.

Puig, J.M (2012). La cultura moral como sistema de prácticas y mundo de valores. En J.M. Puig (coord.) Cultura moral y educación, pp. 87-105. Barcelona: Graó.

Sapon-Shevin, M. (2013). La inclusión real: Una perspectiva de justicia social [Versión en castellano]. Revista de investigación en Educación, 11 (3), pp. 71-85.

Sandel. M. (2020) La tiranía del mérito. ¿Qué ha sido del bien común? España: Debate.

Schlessinger, S. (2018). Reclaiming teacher intellectualism through and for inclusive education, International Journal of Inclusive Education, 22 (3), pp. 268-284. Doi: 10.1080/13603116.2017.1362598/.

Slee, R. (2019). Belonging in an age of exclusion. International Journal of Inclusive Education, 23(9), pp. 909-922. Doi: 10.1080/13603116.2019.1602366/.

Smeed, J. y Carrington, S. (2018). Using accountability data as a catalyst. En J. Harris, S. Carrington y M. Ainscow (eds.) Promoting equity in schools. Collaboration, Inquire and Ethical Leadership (30-44). Devon, UK: Routledge.

Steiner, A. (2018). Prólogo: el estado del desarrollo humano mundial en 2018. En Programa de las Naciones Unidas para el Desarrollo. Índices e indicadores del desarrollo humano. Actualización estadística 2018 (pp. III y IV). Nueva York: PNUD.

Strauss, S. y Ziv, M. (2004). Teaching: ontogenesis, culture and education. CognitiveDevelopment, 19, pp. 451-456. Doi:10.1016/j.cogdev.2004.09.001 
Tedesco, J.C. (2010). La educación en el horizonte 2020. Educación y justicia: el sentido de la educación. $\quad$ Fundación Santillana. En http://www.redage.org/sites/default/files/adjuntos/documento_bsico.pdf /.

Tronto, J. (1987). Más allá de la diferencia de género. Hacia un teoría del cuidado. Sign: Journal of Women in Culture and Society. 12, pp. 1-17.

Tronto. (2017). There Is an Alternative: Homines Curans and the Limits of Neoliberalism. International Journal of Care and Caring, 1(1) pp. 27-43. Doi: 10.1332/239788217X14866281687583.

UNESCO. (2015). Educación 2030. Declaración de Incheon y Marco de Acción. Hacia una educación inclusiva y equitativa de calidad y aprendizaje a lo largo de la vida para todos.

http://www.unesco.org/new/fileadmin/MULTIMEDIA/FIELD/Santiago/pdf/ESPMarco-de-Accion-E2030-aprobado.pdf/.

UNESCO. (2016). Educación 2030. Declaración de Incheon y Marco de Acción para la realización del Objetivo de Desarrollo Sostenible 4. Garantizar una educación inclusiva y equitativa de calidad y promover oportunidades de aprendizaje permanente para todos. En http://unesdoc.unesco.org/images/0024/002456/245656s.pdf .

UNESCO. (2018). Informe de seguimiento de la educación en el mundo 2019. Migración, desplazamiento y educación: construyendo puentes, no muros. París: En https://es.unesco.org/gem-report/node/1878/.

UNESCO. (2017). Una nueva hoja de ruta para el Programa sobre el Hombre y la Biosfera (MAB) y su Red Mundial de Reservas de Biosfera. París: UNESCO. En http://rerb.oapn.es/images/PDF_publicaciones/plan_de_accion_de_lima-20162025.pdf/.

UNESCO. (2020). Towards inclusion in education: status, trends and challenges: the UNESCO Salamanca Statement 25 years on. Paris: UNESCO. En https://unesdoc.unesco.org/ark:/48223/pf0000374246/.

UNESCO/BIE. (2008). Informe de la 48 Conferencia Internacional. Laeducación inclusiva, el camino hacia el futuro, conclusiones y recomendaciones de la $48^{a}$ reunión de la Conferencia Internacional de Educación (CIE). Ginebra: Oficina Internacional de la Educación de la UNESCO. https://unesdoc.unesco.org/ark:/48223/pf0000180629_spa/. 
World Meteorological Organization. (2019). WMO Statement on the state of the Global Climate in 2018. Geneva: WMO. En: https://library.wmo.int/doc_num.php?explnum_id=5789

\section{Notas:}

En adelante emplearemos el término "escuela o educación escolar" para referirnos a la educación formal en sus niveles obligatorios.

${ }^{2}$ Hay cierto acuerdo entre los expertos en considerar la moral como la exigencia humana de responder ante los propios actos y la ética como objeto de debate filosófico en torno a esas cuestiones. Si bien, también es reconocido que ambos conceptos tienen sus límites difusos. En este trabajo optamos por la incorporación del término ética frente a moral por la posibilidad que le atribuimos de enfatizar la visión del ser humano, de las instituciones sociales y/o de la sociedad como actores clave para la transformación de las situaciones de injusticia, en línea con la propuesta de Freire (2009) del ser inacabado: "La inconclusión que se reconoce a sí misma implica necesariamente la inserción del sujeto inacabado en un permanente proceso social de búsqueda (...), la conciencia del inacabamiento nos hizo seres responsables, por eso la eticidad de nuestra presencia en el mundo" (pp. 54 y 55).

${ }^{3}$ No es nuestra intención situar la escuela como la única herramienta para transformar la sociedad. No estamos queriendo situarnos en un escenario cercano al que algunos identificaron como optimismo pedagógico propio de los 60 (Murillo y Krichesky, 2015) y reconocemos el valor de los otros contextos educativos y sociales (Coll, 2013) a la vez que los consideramos indispensables en la tarea. Hablamos por tanto de una escuela que se suma a un proyecto social compartido.

${ }^{4}$ Principalmente circunscribiremos la reflexión a la situación vivida por el alumnado considerado con necesidades educativas especiales (n.e.e.), como ejemplo ilustrativo y paradigmático de las consecuencias sociales que implica nuestro modo de comprender las diferencias entre las personas. 